\title{
AMCoR
}

Asahikawa Medical University Repository http://amcor.asahikawa-med.ac.jp/

Gastrointestinal Endoscopy (2010) 72(6):1303-1307.

A case of early Barrett's adenocarcinoma repeatedly developing multiple metachronous lesions shortly after endoscopic therapy: an analysis for genetic and epigenetic alterations

Watari, Jiro ; Sakurai, Jun ; Morita, Tsuyoshi ; Yamazaki, Takahisa ; Okugawa, Takuya ; Toyoshima, Fumihiko ;

Tanaka, Junji ; Tomita, Toshihiko ; Kim, Yongmin ; Oshima, Tadayuki ; Hori, Kazutoshi ; Moriichi, Kentaro ; Tanabe, Hiroki ; Fujiya, Mikihiro ; Kohgo, Yutaka ; Das, Kiron M. ; Matsumoto, Takayuki ; Miwa, Hiroto 


\section{-Case report-}

A case of early Barrett's adenocarcinoma repeatedly developing multiple metachronous lesions in a short period following endoscopic therapy: an analysis for genetic and epigenetic alterations

A brief title: Barrett's adenocarcinoma repeating metachronous lesions

Jiro Watari, MD, ${ }^{1}$ Jun Sakurai, MD ${ }^{1}$ Tsuyoshi Morita, MD, ${ }^{1}$ Takahisa Yamazaki, MD, ${ }^{1}$

Takuya Okugawa, $\mathrm{MD}^{1}$, Fumihiko Toyoshima, $\mathrm{MD}^{1}$, Junji Tanaka, MD, ${ }^{1}$ Toshihiko Tomita, MD, ${ }^{1}$ Yongmin Kim, MD, ${ }^{1}$ Tadayuki Oshima, MD, ${ }^{1}$ Kazutoshi Hori, MD, ${ }^{1}$ Kentaro Moriichi, MD, ${ }^{2}$ Hiroki Tanabe, MD, ${ }^{2}$ Mikihiro Fujiya, MD, ${ }^{2}$ Yutaka Kohgo, MD, ${ }^{2}$ Kiron M. Das, MD, ${ }^{3}$ Takayuki Matsumoto, MD, ${ }^{1}$ Hiroto Miwa, $\mathrm{MD}^{1}$

${ }^{1}$ Division of Upper Gastroenterology, Department of Internal Medicine, Hyogo College of Medicine, Nishinomiya, Japan; ${ }^{2}$ Division of Gastroenterology and Hematology/Oncology, Department of Medicine, Asahikawa Medical College, Asahikawa, Japan; ${ }^{3}$ Crohn’s and Colitis Center of New Jersey, Division of Gastroenterology and Hepatology, Department of Medicine, UMDNJ-Robert Wood Johnson Medical School, New Brunswick, New Jersey Key words: Barrett’s cancer, Metachronous, Endoscopic treatment, Molecular pathology

All authors have read and approved the final manuscript.

Disclosure: None of the authors have a conflict of interest. Moreover, we have no grants or financial support for this work. Written informed consent was obtained from the patient 
Watari et al. 2

beforehand.

Address Correspondence to: Jiro Watari, MD

Division of Upper Gastroenterology,

Department of Internal Medicine,

Hyogo College of Medicine,

1-1, Mukogawa-cho, Nishinomiya, Hyogo 663-8501, Japan

Tel: +81-798-45-6662, Fax: +81-798-45-6661

E-mail: watarij@hyo-med.ac.jp 


\section{Introduction}

Barrett's esophagus (BE) is a premalignant condition and the most important known risk factor for esophageal adenocarcinoma (EAC) [1]. Although intestinal metaplasia (IM) in $\mathrm{BE}$ mucosa is considered a precursor for EAC, there is a lack of universal agreement regarding the inclusion of IM as a criterion for the diagnosis of BE between the US and Europe [2,3]. We report herein a case that repeatedly developed multiple metachronous cancers at the $\mathrm{BE}$ mucosa without IM after undergoing endoscopic treatment for early EAC. Additionally, we evaluated the chronology of genetic and epigenetic events as well as cellular phenotype, as identified by a monoclonal antibody (mAb) Das-1 related to carcinogenesis that specifically (100\%) reacts with $\mathrm{BE}$ and EAC [4,5].

\section{CASE REPORT}

A 59-year-old man underwent upper gastrointestinal endoscopy as part of his routine medical check-up in April 2009, at which time an elevated lesion in the esophagus was detected. He had a history of polycystic kidney, and he had received regular dialysis treatment, but did not have a family history of malignancies. Upper endoscopy revealed an elevated lesion $1 \mathrm{~cm}$ in diameter in a long-segment BE approximately $12 \mathrm{~cm}$ long. The lesion showed a sharply marginated and irregular surface on chromoendoscopy (Fig. 1). Magnified high-resolution narrow band imaging (NBI) revealed that the microvessels of the tumor surface were irregular, and the diameters and distribution of the vessels were heterogeneous. As early EAC was suspected on the endoscopic findings including NBI and biopsies, cap-assisted endoscopic mucosal resection (c-EMR) was performed after obtaining written informed consent. The resected area was 26 x $21 \mathrm{~mm}$, and complete en bloc resection with 
tumor-free margins was accomplished histopathologically. The histology revealed intramucosal moderately differentiated adenocarcinoma with lymphatic invasion in the muscularis mucosae, and did not demonstrate any findings of IM with goblet cells and dysplastic glands surrounding the malignancy. Follow-up esophagoscopy at 2 months after the initial EMR identified multiple areas of irregular mucosa around the EMR scar, and the biopsy samples were diagnosed histologically as well-differentiated adenocarcinoma (Fig. 2). Thereafter, extensive contact thermal ablation (CTA) with c-EMR was performed on the lesions. The CTA procedure was performed with the lateral of hot biopsy forceps (FD-1L-1, Olympus, Tokyo) with a 40W soft-mode coagulation current (effect 5) with an electrosurgical unit (VIO 300D, ERBE, Germany). Two months later, following additional endoscopic therapy, cancers developed again around the scar (Fig. 3). Finally, the remaining BE was completely eradicated with 7 sessions of EMR and/or extensive ablation therapy within 10 months.

We evaluated the microsatellite instability (MSI), the methylation status, and immunoreactivity using a mAb Das-1 in the cancer and the background mucosa according to our previous reports [4-7]. Both cancer and biopsy materials from the BE mucosa were found to be positive for MSI at the locus of D5S346 and D17S250 among 5 loci investigated [8]. The promoter methylation at the APC gene was present under the two conditions (Fig. 4). However, mAb Das-1 reacted with the cancer area but not with the BE mucosa.

\section{DISCUSSION}

Recent publications by some investigators and groups have reported on short- to long-term data in patients with high-grade intraepithelial neoplasia and early cancer treated by various modalities of endoscopic therapy. During follow-up examinations, metachronous 
neoplasia have been shown to the major problem with endoscopic therapy [9-13]. In the current case, all lesions developed around the scar after endoscopic therapy. Although the reasons why the multiple metachronous lesions became visible adjacent to the treated region in the short term remain unclear, there are a few explanations for the phenomenon. First, it was possible that these lesions were missed at the initial and follow-up endoscopy. However those lesions were not detected even though BE mucosa was observed thoroughly by chromoendoscopy and NBI prior to the initial treatment for EAC. Second, occult cancers that are not detectable at the time of endoscopic therapy may grow within a short period in response to a stimulus such as a growth factor $[14,15]$ during the healing process of the endoscopic treated area. However, no evidence was found of dysplasic glands and IM in 24 biopsy specimens from long-segment BE, including the surrounding mucosa of the tumor. Pech et al. have described that the majority of recurrences or metachronous neoplasia found during follow-up are de novo neoplasias, and that these lesions usually develop due to genetic abnormalities within the remaining BE mucosa and are not influenced by endoscopic therapy [10].

Recently, Liu et al. have shown that DNA content abnormalities occur with equal frequency and extent in metaplastic columnar epithelium of the esophagus without IM compared with metaplastic columnar epithelium with IM [16]. We have previously reported genetic instability and epigenetic abnormalities, including APC, in BE with/without IM [6,7]. Furthermore, it has recently been reported that hypermethylation of APC strongly predict the progression to cancer in patients with BE [17]. These results indicate that EAC patients with genetic instability and APC hypermethlation in the background mucosa may be at a high risk of metachronous lesions. It has been reported that mAb Das-1 may allow early detection of 
metaplastic epithelium of the colonic phenotype in the absence of histological evidence of specialized columnar epithelium [5]. In our case, however, columnar-lined epithelium showed negative staining to this antibody.

Taking previous reports $[16,17]$ and the current case together, EAC patients with genetic instability and epigenetic alterations in the background BE mucosa may be at risk for recurrence even if IM is not observed in the biopsy samples. Thus, BE with those DNA alterations should be completely eradicated and be followed-up more intensively after endoscopic treatment. 


\section{References}

1. Shaheen NJ, Crosby MA, Bozymski EM, et al. Is there publication bias in the reporting of cancer risk in Barrett's esophagus? Gastroenterology 2000;119:333-8.

2. Wang KK, Sampliner RE; Practice Parameters Committee of the American College of Gastroenterology, et al. Updated guidelines 2008 for the diagnosis, surveillance and therapy of Barrett's esophagus. Am J Gastroenterol 2008;103:788-97.

3. Playford RJ. New British Society of Gastroenterology (BSG) guidelines for the diagnosis and management of Barrett's oesophagus. Gut 2006;55:442.

4. Das KM, Prasad I, Garla S, et al. Detection of a shared colon epithelial epitope on Barrett epithelium by a novel monoclonal antibody. Ann Intern Med 1994;120:753-6.

5. Griffel LH, Amenta PS, Das KM. Use of a novel monoclonal antibody in diagnosis of Barrett's esophagus. Dig Dis Sci 2000;45:40-8.

6. Moriichi K, Watari J, Das KM, et al. Effects of Helicobacter pylori infection on genetic instability, the aberrant $\mathrm{CpG}$ island methylation status and the cellular phenotype in Barrett's esophagus in a Japanese population. Int J Cancer 2009;124:1263-9.

7. Watari J, Moriichi K, Tanabe H, et al. Differences in genetic instability and cellular phenotype among Barrett's, cardiac, and gastric intestinal metaplasia in a Japanese population with Helicobacter pylori. Histopathology 2009;55:261-9.

8. Boland CR, Thibodeau SN, Hamilton SR, et al. National Cancer Institute on Workshop on Microsatellite instability for Cancer detection and familial predisposition: development of international criteria for the determination of microsatellite instability in colorectal cancer. Cancer Res 1998;58:5248-57.

9. Ell C, May A, Pech O, et al. Curative endoscopic resection of early esophageal 
adenocarcinomas (Barrett's cancer). Gastrointest Endosc 2007;65:3-10.

10. Pech O, Behrens A, May A, et al. Long-term results and risk factor analysis for recurrence after curative endoscopic therapy in 349 patients with high-grade intraepithelial neoplasia and mucosal adenocarcinoma in Barrett's oesophagus. Gut 2008;57:1200-6.

11. Chennat J, Konda VJ, Ross AS, et al. Complete Barrett's eradication endoscopic mucosal resection: an effective treatment modality for high-grade dysplasia and intramucosal carcinoma--an American single-center experience. Am J Gastroenterol 2009;104:2684-92.

12. Shaheen NJ, Sharma P, Overholt BF, et al. Radiofrequency ablation in Barrett's esophagus with dysplasia. N Engl J Med 2009;360:2277-88.

13. Pouw RE, Wirths K, Eisendrath P, et al. Efficacy of radiofrequency ablation combined with endoscopic resection for Barrett's esophagus with warly neoplasia. Clin Gastroenterol Hepatol 2010;8:23-29.

14. Aldulaimi D, Jankowski J. Barrett's esophagus: an overview of the molecular biology. Dis Esophagus 1999;12:177-80.

15. Brito MJ, Filipe MI, Linehan J, et al. Association of transforming growth factor alpha (TGFA) and its precursors with malignant change in Barrett's epithelium: biological and clinical variables. Int J Cancer 1995;60:27-32.

16. Liu W, Hahn H, Odze RD, et al. Metaplastic esophageal columnar epithelium without goblet cells shows DNA content abnormalities similar to goblet cell-containing epithelium. Am J Gastroenterol 2009;104:816-24.

17. Wang JS, Guo M, Montgomery EA, et al. DNA promoter hypermethylation of p16 and APC predicts neoplastic progression in Barrett's esophagus. Am J Gastroenterol 2009;104:2153-60. 
Watari et al. 9

\section{Figure legends}

Figure 1. A, Chromoendoscopy showed a sharply marginated elevated Barrett's adenocarcinoma. B, With Magnified high-resolution NBI, irregular vascular pattern with abnormal blood vessels was found. C, The resected specimen revealed intramucosal moderately differentiated adenocarcinoma with lymphatic invasion in the muscularis mucosae. Figure 2. A, Two months after the initial endoscopic mucosal resection (EMR), multiple areas of irregular mucosa (arrows) around the EMR scar (asterisk) was identified. The biopsy samples obtained from irregular mucosa revealed well-differentiated to papillary adenocarcinoma. B, The EMR specimens showed intramucosal papillary adnocarcinoma.

Figure 3. A, Follow-up esophagoscopy with NBI at 2 months after the second EMR identified multiple brownish irregular mucosa (arrowheads) around the endoscopic treatment (asterisk). B, The biopsy samples confirmed well-differentiated adenocarcinoma.

Figure 4. A, Microsatellite instabilities on D5S346 and D17S250 were seen as an unequivocal extra peak shift (asterisks) in both Barrett’s esophagus (BE) and esophageal adenocarcinoma (EAC) compared with normal gastric mucosa (control). B, The promoter methylation of APC was present in both BE and EAC (arrows). 
A
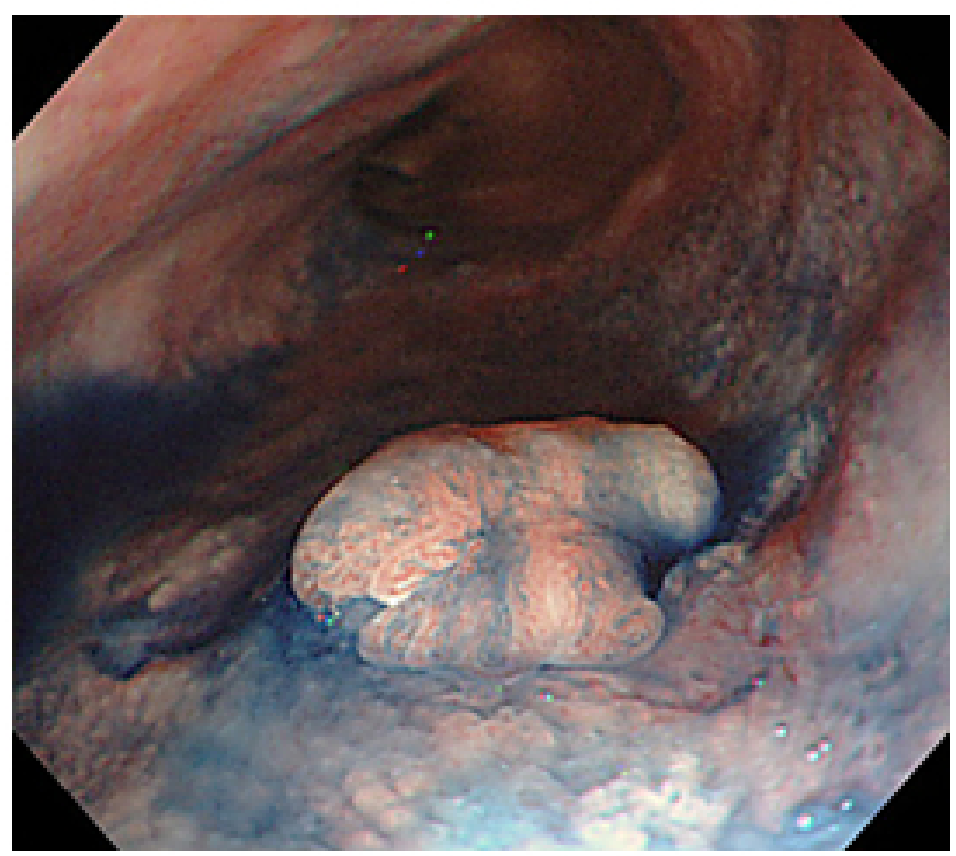

C

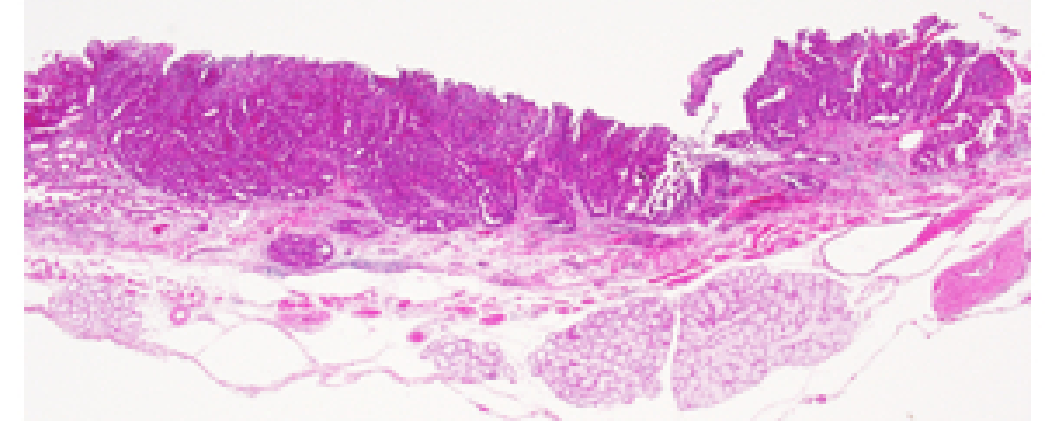

B

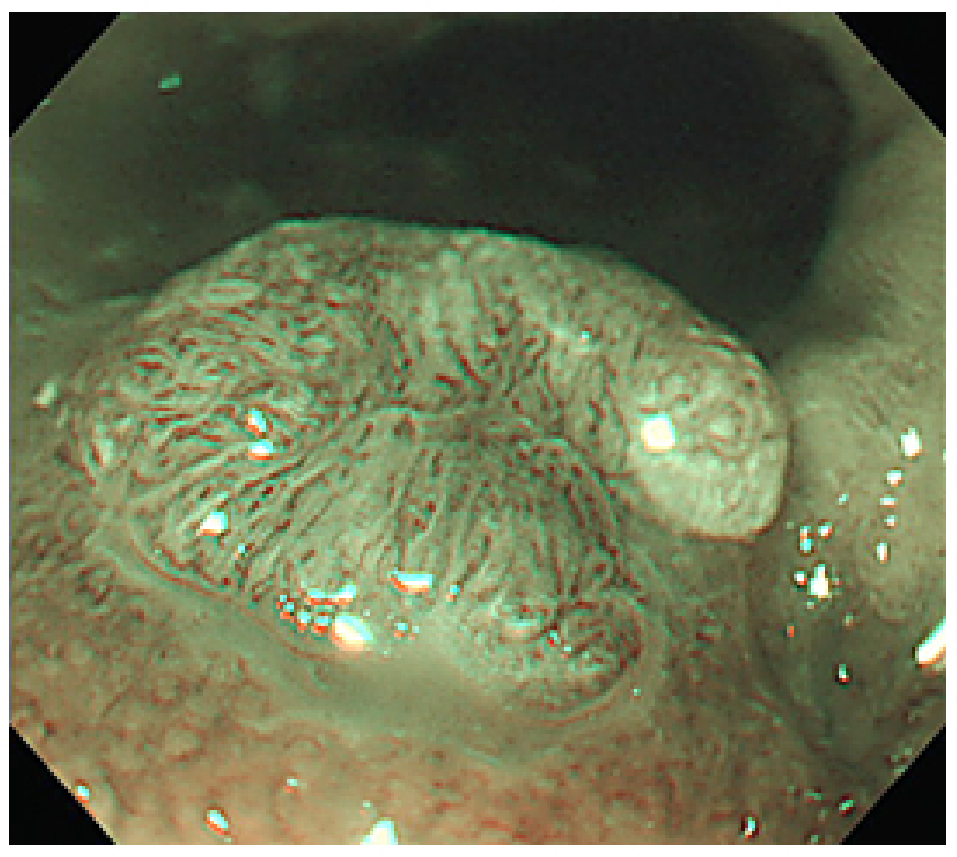

Figure 1 

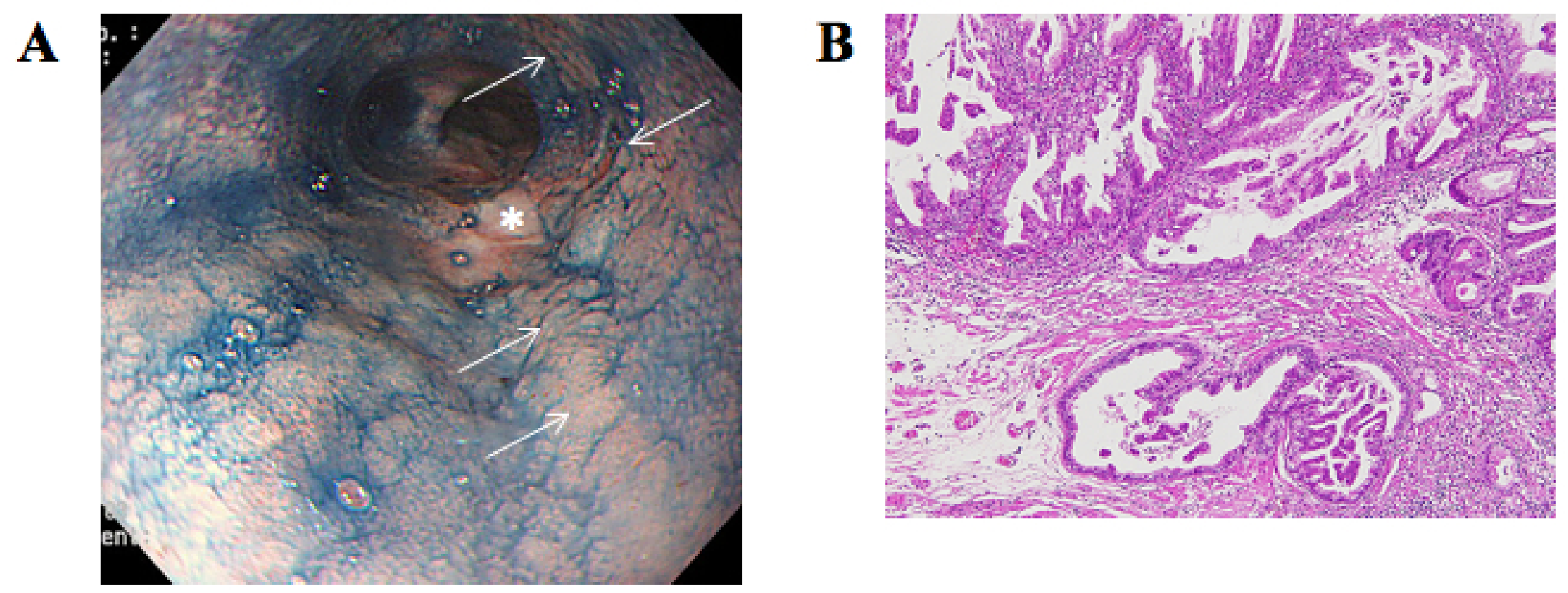

Figure 2 



Figure 3 
B

APC


Figure 4 